\title{
An fNIRS-Based Study on Prefrontal Cortex Activity during a Virtual Shopping Test with Different Task Difficulties in Brain-Damaged Patients
}

\author{
Sayaka Okahashi',2*, Hokuto Mizumoto², Akiko Komae ${ }^{3}$, Katsuhiro Ueno ${ }^{3}$, \\ Masato Yokoyama4, Akinori Nagano5, Keiko Seki6, Toshiko Futaki' ${ }^{1}$, Zhiwei Luo ${ }^{2}$ \\ ${ }^{1}$ Division of Occupational Therapy, Department of Human Health Sciences, Graduate School of Medicine, Kyoto \\ University, Kyoto, Japan \\ ${ }^{2}$ Department of Computational Science, Graduate School of System Informatics, Kobe University, Kobe, Japan \\ ${ }^{3}$ Department of Rehabilitation Medicine, Nishi Memorial Port Island Rehabilitation Hospital, Kobe, Japan \\ ${ }^{4}$ Department of Neurology, Nishi Memorial Port Island Rehabilitation Hospital, Kobe, Japan \\ ${ }^{5}$ Faculty of Sport and Health Science, Ritsumeikan University, Shiga, Japan \\ ${ }^{6}$ Department of Rehabilitation Science, Graduate School of Health Sciences, Kobe University, Kobe, Japan \\ Email: " ${ }^{*}$ kahashi.sayaka.7c@kyoto-u.ac.jp, hokuto@cs11.cs.kobe-u.ac.jp, nmpr-riha@koyukai.or.jp, \\ aknr-ngn@fc.ritsumei.ac.jp, ksekikb@yahoo.co.jp, futaki.toshiko.2c@kyoto-u.ac.jp, luo@gold.kobe-u.ac.jp
}

Received 20 April 2014; revised 31 May 2014; accepted 16 June 2014

Copyright (C) 2014 by authors and Scientific Research Publishing Inc.

This work is licensed under the Creative Commons Attribution International License (CC BY).

http://creativecommons.org/licenses/by/4.0/

(c) () Open Access

\section{Abstract}

We developed a Virtual Shopping Test with three different task levels for assessment of daily cognitive function using virtual reality technology. The objective of present study was to investigate the difference on task performance, brain activation and subjective assessment in relation to the difficulty levels of the tasks. Subjects were asked to buy specific 2 items in Task 1, 4 items in Task 2 , and 6 items in Task 3 at a virtual mall. The tasks and questionnaires were conducted on 10 convalescent brain-damaged patients and 6 healthy young adults. Hemodynamic changes in the prefrontal cortex (PFC) during activation due to the tasks were examined using functional near-infrared spectroscopy. As the result, the mean total time was significantly longer for the patients than for healthy subjects. PFC showed a greater response for related Task 2 than Task 1 in shopping and moving phase in patient group. The patients evaluated Tasks 1 and 2 are more difficult and bring more psychological load than healthy adults subjectively. That is, although the healthy adults did not show large difference in their task performances as well as PFC responses, they can evaluate the differences between three task levels, subjectively, while which could not be

\footnotetext{
"Corresponding author.
}

How to cite this paper: Okahashi, S., et al. (2014) An fNIRS-Based Study on Prefrontal Cortex Activity during a Virtual Shopping Test with Different Task Difficulties in Brain-Damaged Patients. Journal of Behavioral and Brain Science, 4, $247-255$. 
for the patients means that patients could not distinguish the difference of the tasks, subjectively. The results suggest that 4-item shopping task might be enough difficulty level that causes brain activation for the brain-damaged patients.

\title{
Keywords
}

\author{
Cognitive Assessment, Brain Activation, Virtual Reality, Task Difficulty, Rehabilitation
}

\section{Introduction}

Higher-order cognitive dysfunction about memory, attention, language and so on due to brain damage (e.g., traumatic brain injury, stroke) and aging causes many difficulties in their everyday life. In modern society, the population of elderly people having various physical and mental diseases is increasing. The development of medical care and rehabilitation for the dysfunction is necessary. Especially, the patients with higher-order cognitive dysfunction demonstrate difficulties in activities of daily living (ADL) and instrumental activities of daily living (IADL) [1] [2]. Therefore, in order to evaluate the cognitive functions, the environment that is almost similar to everyday life condition is important. Considering this effect, recently, the virtual reality (VR) techniques have been used.

In cognitive rehabilitation, the exercises with appropriate difficulty level for individual may increase the patients' motivation and produce good results. Clinically, rehabilitation therapists evaluate each patient's ability and try to provide him/her with the specific appropriate tasks. However, it is sometimes difficult to set the appropriate task level because of less evidence about the effectiveness [3]. So far, various techniques using VR have been proposed in cognitive rehabilitation [4]-[6]. However, they did not discuss on task difficulty level related to brain activation sufficiently.

We developed a Virtual Shopping Test (VST) for realistic assessment of cognitive function using VR technology [7]. In this research we modified it so that the revised version of VST (VST-R) had three different task difficulty levels. The system can also output event signals synchronized with users' PC operation. We could use the signals in analyzing event related to brain activation. In present study we used our VST-R with functional near-infrared spectroscopy (fNIRS) for examination of prefrontal cortex activity during the test performance.

fNIRS is a non-invasive and non-restrictive technique that allows the measurement of blood oxygenation changes related to cerebral activation [8] [9]. Unlike other modern neuroimaging technique such as functional magnetic resonance imaging (fMRI) and positron emission tomography (PET), fNIRS has advantages on the daily clinical therapy situation. fNIRS has been used to study development and recovery of physical/cognitive function in healthy people and brain-damaged patients in wide age range [10] [11].

The objective of this study was to investigate the difference on task performance, brain activation and subjective assessment in relation to the task difficulty levels. Subjects were asked to buy specific 2 items, 4 items, and 6 items in a different virtual mall in each task. These tasks and questionnaires were conducted on convalescent brain-damaged patients and healthy young adults. Hemodynamic changes in the prefrontal cortex (PFC) during activation due to the tasks were examined using fNIRS. It is reported that executive function and attention were related to PFC activates [12]. We hypothesized that each level of task would cause different brain activation and subjective assessment, and the pattern would be also different between the two groups.

\section{Materials and Methods}

\subsection{Participants}

Ten brain-damaged in patients with cognitive dysfunction in the convalescence rehabilitation ward ( 5 males, 5 females, mean age $61.2 \pm 16.2$ years) and six young healthy subjects (4 males, 2 females, mean age $23.2 \pm 1.0$ years) participated in this study. For the patients, the participation criteria were as follows: 1 ) cognitive ability to understand how to operate a touch screen and 2) physical ability to reach and touch the screen by their uninvolved upper limbs. The study exclusion criteria were as follows: 1) severe aphasia, and 2) severe unilateral spatial neglect. All participants received written and verbal information about the study and gave written informed 
consent. The protocol of the study was approved by Kobe university graduate school of system informatics ethic committee and Nishi memorial port island rehabilitation hospital ethic committee.

\subsection{The Virtual Shopping Test-Revised (VST-R) with fNIRS}

The hardware system included a personal computer, a touch screen (LCD-MF222FBR-T, I-O DATA) and 16channel OEG-16 fNIRS system (Spectratech Co., Yokohama, Japan). Figure 1 shows the overall setup of the experimental device. The virtual environment was developed with Metasequoia and Open GL. In this program, visual environment was made up of a Japanese shopping mall with 20 shops and a train station. An audio environment of natural sound of the shopping mall was also provided. By touching the bottom of the screen, users could move forward and turn back in the virtual shopping mall, entering a shop and buying an item. Two hint buttons (e.g., List and Bag) were provided to allow users to view some hints during the shopping task. The operation of buttons was recorded automatically, and outputted as a log file after finishing the test. In addition, this system output event signals when users enter/exit the shop, buy an item and check a purchase list. fNIRS data was recorded with the signals.

\subsubsection{Task Setting on VST-R}

We construct a virtual shopping mall in a virtual space, and set up three different tasks with different difficulties. Task 1 asks subjects to buy 2 specific items (a pudding and an AAA-sized battery), Task 2 asks them to buy 4 specific items (a tomato, rubber boots, an alarm clock and a teacup), and Task 3 asks them to buy 6 specific items (an eraser, plum liqueur, incense sticks, building blocks, 80-yen stamp and a glove). A visual shopping mall on each task had 20 shops and a train station, but the shop arrangement in a mall was different between tasks. Subjects must search the shops that sell specific items and select the target item out of six items inside a shop. Figure 2 shows a basic VST-R screen and Figure 3 shows the virtual shopping mall map on Task 3.

\subsubsection{Experimental Procedure on VST-R}

The subjects were first asked to memorize the specific shopping items while looking at the shopping list for 10 seconds. Then, subjects were allowed to plan their shopping routes that they thought would be the most efficient by filling in a blank map sheet with a pencil. They were then asked to perform buying the specific items as quickly and efficiently as possible, while minimizing the use of hints as much as possible. They were allowed to refer to their planning using the blank map during the VST at any time.

\subsubsection{Outcome Variables on VST-R}

VST-R had eight outcome variables: the number of times subjects used each button (Bag use, List use, Forward

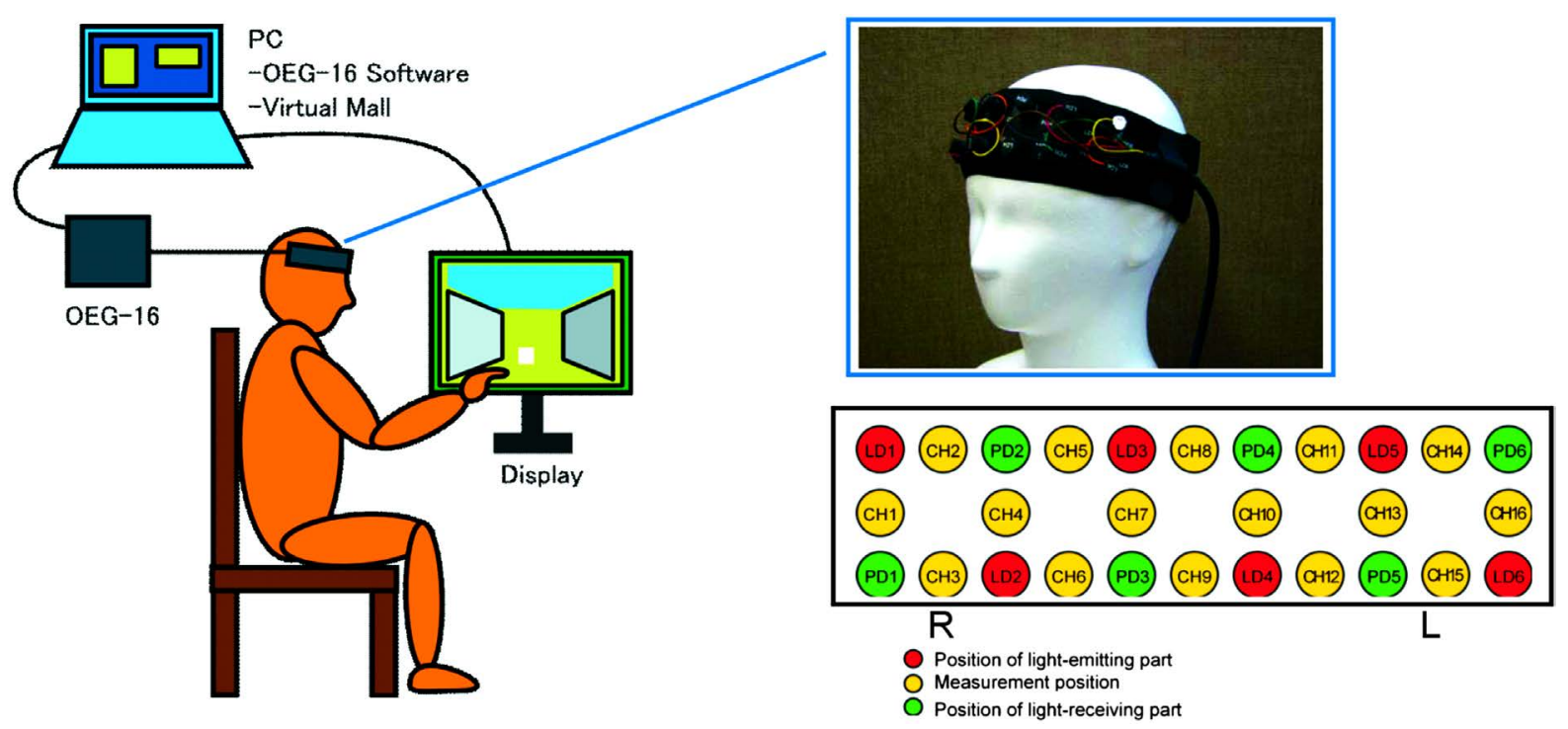

Figure 1. Experimental system and fNIRS channel arrangement on the forehead. 


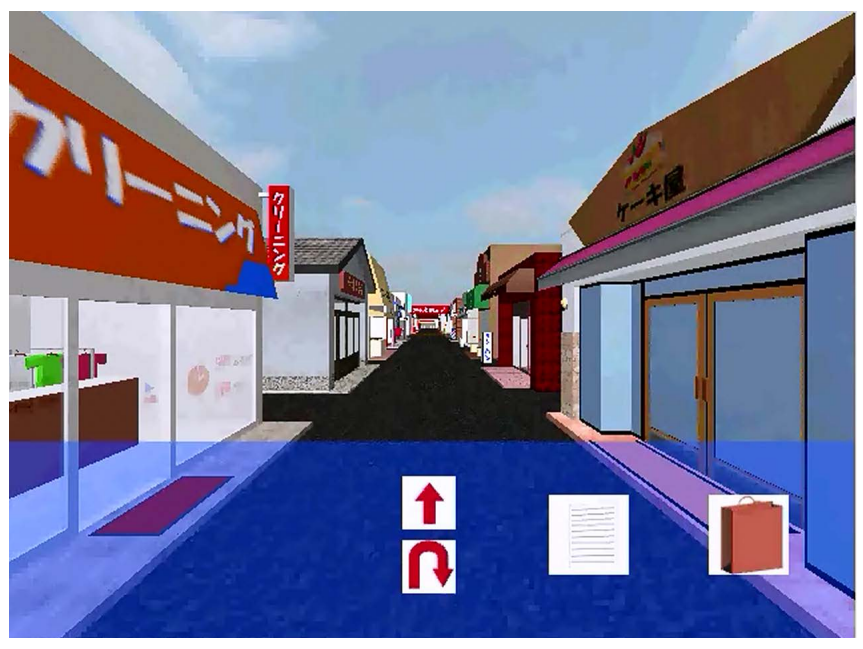

Figure 2. An example of a basic screen on VST-R.

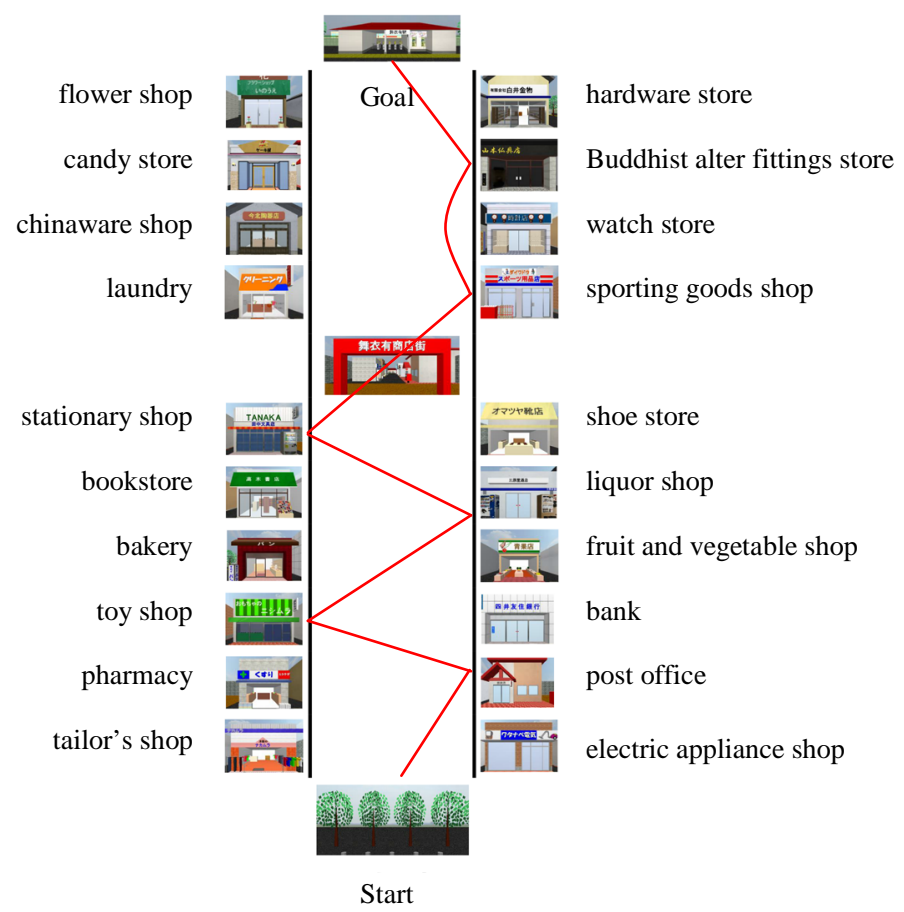

Figure 3. Map of a virtual shopping mall on Task 3. There were twenty shops and a station in the virtual Japanese shopping mall. The bottom was the start point, while the top was the goal. The red route showed the most efficient shopping order.

movement, and Reverse movement), the number of items bought correctly (Correct purchases), Total time, Time in shops, Time on road. They could be calculated from the recording data.

\subsection{4. fNIRS}

A 16-channel OEG-16 fNIRS system was used at a sampling time of 0.65 seconds and measured the intensity of light detected at two wavelengths, 770 and $840 \mathrm{~nm}$ and calculated the change in optical density. It then calculated the changes in oxygenated hemoglobin concentration [oxyHb], deoxygenated hemoglobin concentration [deoxyHb] and total hemoglobin concentration [totalHb] based on at the Beer-Lambert approach. The emission and detection probes were bilaterally attached to the forehead of each subject. The detection probes were set at 
Fp1 and Fp2 corresponding to the international 10 - 20 system of electrode placement with the emission probes. Channels covered the left and right PFC.

\subsection{Procedure}

All participants were administered VST-R in the order of Task 1, 2, and 3 and questionnaires concerning subjective task difficulty after each task. The questionnaire consisted of 3 questions concerning the degree of task difficulty, made efforts, and psychological load. Each answer was rated on a five-point scale (1 - 5). Higher scores indicated higher load task. In addition, Patients were administered seven conventional neuropsychological tests and two questionnaires. General cognitive level was evaluated by Mini-Mental State Examination (MMSE). Attention was evaluated by symbol Digit Modalities Test (SDMT) [13] and Simple Reaction Time Task (SRT) [14]. Regarding visual inattention, the presence of USN was assessed by Star and Letter Cancellation Task [15]. Everyday memory was assessed by Rivermead Behavioural Memory Test (RBMT) [16] and Everyday Memory Checklist (EMC) [17]. Executive function was evaluated by Zoo Map Test and the Dysexecutive Questionnaire (DEX) [18]. All tests were finished within one month before and after VST-R execution.

\subsection{Data Analysis}

For fNIRS data analysis, we reset the data at the start of encoding words and VST performance. We used the low pass filter (cutoff frequency $0.05 \mathrm{~Hz}$ and attenuation slope $40 \mathrm{~dB} / \mathrm{Oct}$ ). We used Ch4-6 data as the right lateral prefrontal cortex (rPFC), Ch7-10 data as the medial prefrontal cortex (mPFC) and Ch11-13 data as the left lateral prefrontal cortex (lPFC). The average change in [oxyHb] of each area was calculated for memorizing phase: while subjects memorize shopping items, shopping phase: while they were in a shop, and moving phase: while they were on a road. Task difficulty level (Task 1, Task 2, Task 3 ) $\times$ Phase (memorizing, shopping, moving) $\times$ Group (patient, healthy) analysis of variance (ANOVA) was conducted on the change in [oxyHb] in each area of PFC. For behavioural data and subjective assessment analysis, Task difficulty level (Task 1, Task 2, Task 3) $\times$ Group (patient, healthy) ANOVA was conducted. Descriptive statistics of performance in all evaluation items were also performed in this study.

\section{Results}

\subsection{Characteristics in Patient Group}

The patients' characteristics and cognitive assessment data were presented in Table 1. The patients included some forms of brain damage experienced within six months from onset. Nine patients of them were over the age of fifty. They all had difficulties in their instrumental activity of daily living. The cognitive dysfunction was related to more than one aspects of ability: attention, memory, and executive function.

\subsection{Behavioural Data}

The comparison of VST-R performance between two groups was presented in Table 2. All the ten patients accomplished Task 1, all patients except ID 5could do Task 2, and all patients except ID 4 and 5could do Task 3. The Patient (ID 4) who needed longest time on Task 1 lost her way in the mall and bought the same item twice. The Patient (ID 5) did not complete Task 2 by using hints and a map efficiently and needed some assist by a tester. The number of purchases was not sufficient or too much on each task for some patients. The mean time required was longer for the patients than for healthy subjects in all tasks. The results of ANOVA showed a significant main effect of Task difficulty level $(\mathrm{p}<0.01)$ and Group $(\mathrm{p}<0.01)$ on the time required (e.g., Total time/Time on road/Time in shop), but no interaction except for the significant Task difficulty level $\times$ Group interaction on Time in shop $(\mathrm{p}<0.05)$. Total time and Time on road were longer on Task 3 than on Task $1(\mathrm{p}<$ 0.01). Time in shop was longer in patient group than in healthy group on Task 2 and $3(\mathrm{p}<0.01)$. However, there was no main effect and no interaction on other variables.

\section{3. fNIRS Data}

Figure 4 illustrated mean changes in [oxyHb] $(\Delta[\mathrm{oxyH}])$ in each memorizing, shopping and moving phase in patient group (see the upper three charts) and healthy group (see the lower three charts). 
Table 1. Patients' characteristics and cognitive assessment data.

\begin{tabular}{|c|c|c|c|c|c|c|c|c|c|c|}
\hline Patient ID & 1 & 2 & 3 & 4 & 5 & 6 & 7 & 8 & 9 & 10 \\
\hline Age & 58 & 64 & 23 & 81 & 59 & 76 & 73 & 66 & 51 & 61 \\
\hline Gender & M & M & $\mathrm{F}$ & $\mathrm{F}$ & M & M & $\mathrm{F}$ & $\mathrm{F}$ & M & $\mathrm{F}$ \\
\hline Diagnosis & $\mathrm{E}$ & CVA & E & TBI & CVA & CVA & TBI & CVA & CVA & CVA \\
\hline Duration of disease (days) & 89 & 157 & 172 & 143 & 55 & 42 & 56 & 101 & 87 & 64 \\
\hline Duration of education (years) & 16 & 15 & 16 & 13 & 16 & 16 & 12 & 12 & 12 & 12 \\
\hline FIM/126 & 126 & 86 & 114 & 111 & 103 & 109 & 120 & 96 & 109 & 125 \\
\hline \multicolumn{11}{|l|}{ General cognitive ability } \\
\hline MMSE/30 & 28 & 26 & 30 & 29 & 27 & 25 & 27 & 26 & 30 & 29 \\
\hline \multicolumn{11}{|l|}{ Attention } \\
\hline SDMT (\%) & 42 & 30 & 33.6 & 17 & 25 & 30 & 25 & 21 & 35 & 31 \\
\hline SRT: correct rate (\%) & 100 & 93.8 & 68.8 & 93.8 & 100 & 88.8 & 76.3 & 62.5 & 100 & 96.3 \\
\hline Star cancellation task/54 & 54 & 52 & 54 & 51 & 64 & 54 & 54 & 54 & 54 & 54 \\
\hline Letter cancellation task/40 & 37 & 38 & 37 & 35 & 35 & 39 & 38 & 39 & 40 & 39 \\
\hline \multicolumn{11}{|l|}{ Memory } \\
\hline RBMT: standard profile score/24 & 20 & 22 & 16 & 14 & 11 & 12 & 9 & 15 & 22 & 16 \\
\hline EMC/39 & 12 & 13 & 2 & 25 & 4 & 19 & 12 & 12 & 4 & 5 \\
\hline \multicolumn{11}{|l|}{ Executive function } \\
\hline Zoo Map Test: the $1^{\text {st }}$ trial/8 & 8 & 6 & 5 & 0 & 5 & 8 & 4 & 7 & 8 & 1 \\
\hline Zoo Map Test: the $2^{\text {nd }}$ trial $/ 8$ & 8 & 8 & 5 & 4 & 8 & 8 & 4 & 8 & 8 & 8 \\
\hline $\mathrm{DEX} / 80$ & 4 & 19 & 41 & 1 & 34 & 1 & 8 & 17 & 5 & 17 \\
\hline
\end{tabular}

M: male, F: female, E: Encephalitis, CVA: cerebrovascular accident, TBI: traumatic brain injury, FIM: Functional Independence Measure.

Table 2. Comparison of VST-R performance between patient group and healthy group.

\begin{tabular}{ccccccc}
\hline & \multicolumn{2}{c}{ Task 1} & \multicolumn{2}{c}{ Task 2} & Task 3 \\
\hline $\begin{array}{c}\text { VST-R } \\
\text { outcome variables }\end{array}$ & Patient group & Healthy group & Patient group & Healthy group & Patient group & Healthy group \\
\hline & $(\mathrm{n}=10)$ & $(\mathrm{n}=6)$ & $(\mathrm{n}=9)$ & $(\mathrm{n}=6)$ & $(\mathrm{n}=8)$ & $(\mathrm{n}=6)$ \\
\hline Bag use & $0(0-1)$ & $0(0-0)$ & $0(0-0)$ & $0(0-0)$ & $0(0-3)$ & $0(0-0)$ \\
List use & $0(0-2)$ & $0(0-0)$ & $2(0-4)$ & $0(0-0)$ & $1.5(0-17)$ & $0(0-0)$ \\
Forward movement & $12(12-26)$ & $12(12-12)$ & $12(12-30)$ & $12(12-12)$ & $12(12-26)$ & $12(12-12)$ \\
Reverse movement & $0(0-4)$ & $0(0-0)$ & $0(0-4)$ & $0(0-0)$ & $1(0-7)$ & $0(0-0)$ \\
Correct purchases & $2(1-3)$ & $2(2-2)$ & $4(3-4)$ & $4(4-4)$ & $6(5-6)$ & $6(5-6)$ \\
Total Time $[\mathrm{s}]$ & $149(84-552)$ & $90(71-114)$ & $211(109-328)$ & $107(98-142)$ & $257(136-647)$ & $137.5(103-151)$ \\
Time in shops $[\mathrm{s}]$ & $19.5(14-38)$ & $12(11-12)$ & $34(25-48)$ & $22.5(22-25)$ & $55.5(36-101)$ & $32(29-39)$ \\
Time on road $[\mathrm{s}]$ & $126.5(65-519)$ & $78.5(69-102)$ & $176(84-285)$ & $85(73-118)$ & $198(100-546)$ & $102(74-117)$ \\
\hline
\end{tabular}

Results show the median (the minimum-the maximum).

We analyzed seven patients' data (except ID 3, 4 and 5) in consideration of little artifact and old age range. In patient group, $\Delta[\mathrm{oxyHb}]$ decreased on Task 1 and 2, and increased on Task 3 in memorizing phase, while $\Delta[\mathrm{oxyHb}]$ decreased on Task 1and increased on Task 2 and 3 in shopping and moving phase. In healthy group, $\Delta[\mathrm{oxyHb}]$ decreased on Task 1 and increased on Task 2 and 3 in memorizing phase, while it decreased on all tasks in shopping and moving phases.

Statistical analyses on $\Delta[0 x y H b]$ were conducted in each area of PFC separately. The results of ANOVAshowed the significant Phase $\times$ Group interaction $(\mathrm{p}<0.05)$, Task difficulty level $\times$ Group interaction $(\mathrm{p}<0.05)$, 
and Task difficulty level $\times$ Phase $\times$ Group interaction $(\mathrm{p}<0.01)$ in lPFC. $\Delta$ [oxyHb] in IPFC increased more in shopping and moving phases on Task 2 for the patients than for healthy subjects significantly $(\mathrm{p}<0.01)$. There was also the significant Task difficulty level $\times$ Phase $\times$ Group interaction $(\mathrm{p}<0.05)$ in mPFC. $\Delta[0 x y H b]$ in $\mathrm{mPFC}$ increased more in shopping phase on Task 2 for the patients than for healthy subjects significantly $(\mathrm{p}<$ 0.01). However, there was no main effect and no interaction in rPFC.

\subsection{Subjective Assessment}

The comparison of subjective assessment between two groups was presented in Table 3. Higher scores indicated higher load task. All the three assessment items tended to be higher for the patients than for healthy subjects. The results of ANOVA showed significant main effect of Task difficulty level $(\mathrm{p}<0.01)$ and Group $(\mathrm{p}<0.05)$ on subjective task difficulty, but no interaction. The mean score was higher on Task 2 and 3 than on Task 1 (p $<$ 0.01). It showed also significant main effect of Task difficulty level $(\mathrm{p}<0.01)$ and Group $(\mathrm{p}<0.01)$ on subjective made effort, but no interaction. The mean score was higher on Task 2 and 3 than on Task 1 ( $p<0.01$ ), and on Task 3 than on Task $2(\mathrm{p}<0.05)$. There were a significant main effect of Task difficulty level $(\mathrm{p}<0.01)$ and the Task difficulty level $\times$ Group interaction $(\mathrm{p}<0.01)$ on subjective psychological load. The patients felt more psychological load than healthy subjects on Task 1 and 2 significantly $(\mathrm{p}<0.01)$. Healthy people felt more psychological load on Task 3 than on Task 1/2, and on Task 2 than on Task 1 significantly ( $<<0.01)$.

\section{Discussion}

The objective of this study was to investigate the difference on task performance, brain activation and subjective assessment in relation to the different task difficulty levels in convalescent brain-damaged patients and healthy young adults.
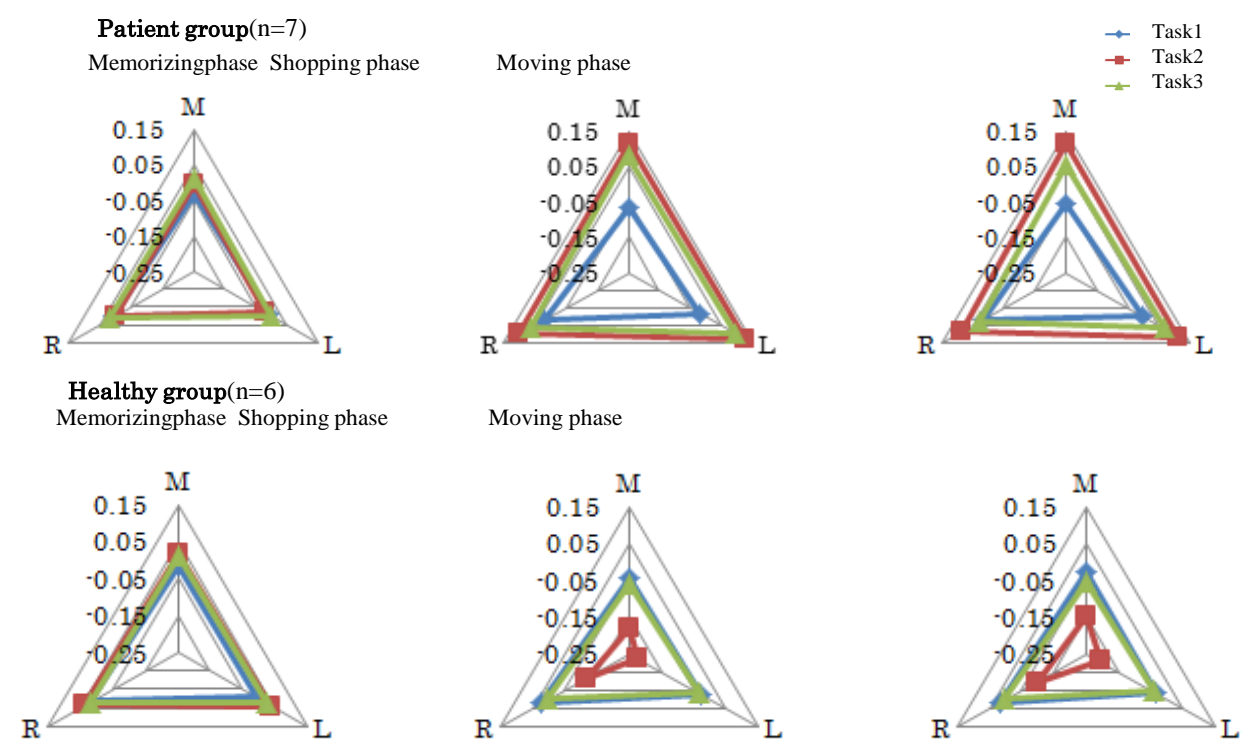

Figure 4. Mean changes in the concentration of oxygenated hemoglobin in each phase.

Table 3. Comparison of subjective assessment between patient group and healthy group.

\begin{tabular}{ccccccc}
\hline & \multicolumn{2}{c}{ Task 1 } & \multicolumn{2}{c}{ Task 2 } & \multicolumn{2}{c}{ Task 3 } \\
\hline Question & Patient group & Healthy group & Patient group & Healthy group & Patient group & Healthy group \\
& $(\mathrm{n}=10)$ & $(\mathrm{n}=6)$ & $(\mathrm{n}=9)$ & $(\mathrm{n}=6)$ & $(\mathrm{n}=8)$ & $(\mathrm{n}=6)$ \\
Task difficulty & $3(1-4)$ & $1(1-2)$ & $4(2-5)$ & $2(2-4)$ & $4(2-5)$ & $4(3-4)$ \\
Made efforts & $2.5(1-4)$ & $1(1-2)$ & $4(2-5)$ & $2(2-3)$ & $4(3-5)$ & $4(2-5)$ \\
Psychological load & $2.5(1-4)$ & $1(1-2)$ & $3.5(1-5)$ & $2(1-4)$ & $3.5(1-4)$ & $3(1-5)$ \\
\hline
\end{tabular}

Results show the median (the minimum-the maximum). 
Firstly, with regard to VST-R performance, one/two patients could not accomplish Task 2/Task 3 respectively. Some patients made mistakes to buy less or more shopping items than directions prescribed beforehand. We considered these patients should start exercise from this difficulty level. The patient (ID 4) aged 81 might need to time to accustom herself to the virtual mall and its operation. In comparison between two groups, the mean time required for the performance was significant longer for the patients than for healthy subjects. The results was consistent with the findings of previous our study in comparison between patient and age-matched control groups [7]. We considered that these variables including the number of purchases on VST-R have important role on cognitive assessment in brain-damaged patients. On the other hand, some times of list use were regarded as natural behaviour, and it would be possible to use VST-R in memory rehabilitation with useful compensation strategy.

Secondly, with regard to brain activation, there was no significant difference on $\Delta[\mathrm{oxyHb}]$ on all tasks in memorizing phase between two groups, while $\Delta[\mathrm{oxyHb}]$ increased more in shopping and/or moving phases on Task 2 in the left and medial PFC for patient group than for healthy group significantly. The pattern of brain activation was different between two groups on Task 2 especially. Generally, it tended that $\Delta[\mathrm{oxyHb}]$ increased in patient group and decreased in healthy group during VST-R performance (e.g., shopping and moving phases) than memorizing phase of shopping items at the beginning of the test. One of the reason for that was young healthy adults were used to use PC in their school life and did not need much effort to operate it, while brain-damaged patients with cognitive dysfunction in the convalescence rehabilitation ward (mean age 61.2 years) were not familiar with PC. The results also suggest that Task 2 and 3 might be enough difficulty level causes brain activation for the brain-damaged patients in this study. Since it was so small sample size, we needed to amass a lot of data and investigate brain activation in relation to task level in detail in our next study. In addition, we need to examine whole hemodynamic changes about not only [oxyHb] but also [deoxyHb] and [totalHb] for each subject based on the previous fNIRS research [19].

Finally, with regard to subjective assessment, the patients evaluated all tasks more difficult and made more effort than healthy people. The patients had more psychological load on Task 1 and 2 (2-item and 4-item shopping task) than healthy adults subjectively. In addition, although the healthy adults can evaluate the differences between three task levels, the patients could not distinguish the difference, subjectively. We consider that therapists should take patients' subjective mental load into consideration, it might be important to check the changes in the exercise phase for more effective rehabilitation.

The results suggest that, although the healthy adults did not show large difference in their task performances as well as PFC responses for different task levels, they can evaluate the differences between three task levels, subjectively, while which could not be for the patients, means that patients could not distinguish the difference of the tasks, subjectively. VST-R is applicable to convalescent patients with cognitive dysfunction in cognitive rehabilitation, and 4-item/6-item shopping task performance causes their PFC activation especially in shopping and moving phases. 2-item shopping task would be applied clinically, taking their behave errors and mental load into consideration.

\section{Conclusion}

This study applied virtual shopping with three different difficulty levels to investigate the difference on task performance, brain activation and subjective assessment in two groups of convalescent brain-damaged patients and healthy young adults. The results suggest that, the two groups show not only different task performances but also different brain activation patterns as well as different subjective assessments during the increasing of the task difficulties as studied detailedly in above discussion. The result is important for our next stage to set more effective task level for the patients in their rehabilitation phase, which may not only depend on their subjective assessment but also more scientific evidences, such as the brain activations and/or task performances.

\section{Acknowledgements}

We express our appreciation to the subjects who participated in this study. The authors are grateful to Yohei Mori, O. T., Kyosuke Nishizawa, O. T., Fumiko Tsuzuki, S. T., for clinical data collection. This research was supported by KAKENHI; Grant-in-Aid for Scientific Research (C) (24500643) and Osaka Gas Group Welfare Foundation. 


\section{References}

[1] Fortin, S., Godbout, L. and Braun, C.M. (2003) Cognitive Structure of Executive Deficits in Frontally Lesioned Head Trauma Patients Performing Activities of Daily Living. Cortex, 39, 273-291. http://dx.doi.org/10.1016/S0010-9452(08)70109-6

[2] Chevignard, M., Pillon, B., Pradat-Diehl, P., Taillefer, C., Rousseau, S., Le Bras, C. and Dubois, B. (2000) An Ecological Approach to Planning Dysfunction: Script Execution. Cortex, 36, 649-669. http://dx.doi.org/10.1016/S0010-9452(08)70543-4

[3] Cicerone, K.D., Langenbahn, D.M., Braden, C., Malec, J.F., Kalmar, K., Fraas, M., Felicetti, T., Laatsch, L., Harley, J.P., Bergquist, T., Azulay, J., Cantor, J. and Ashman, T. (2011) Evidence-Based Cognitive Rehabilitation: Updated Review of the Literature from 2003 through 2008. Archives of Physical Medicine and Rehabilitation, 92, 519-530. http://dx.doi.org/10.1016/j.apmr.2010.11.015

[4] Kang, Y., Ku, J., Han, K., Kim, S., Yu, T., Lee, J., and Park, C. (2008) Development and Clinical Trial of Virtual Reality-Based Cognitive Assessment in People with Stroke: Preliminary Study. CyberPsychology \& Behavior, 11, 329-339. http://dx.doi.org/10.1089/cpb.2007.0116

[5] Knight, R.G., Titov, N. and Crawford, M. (2006) The Effects of Distraction on Prospective Remembering Following Traumatic Brain Injury Assessed in a Simulated Naturalistic Environment. International Psychogeriatrics, 12, 8-16. http://dx.doi.org/10.1017/S1041610207005923

[6] Zhang, L., Abreu, B.C., Seale, G.S., Masel, B., Christiansen, C.H. and Ottenbacher, K.J. (2003) A Virtual Reality Environment for Evaluation of a Daily Living Skill in Brain Injury Rehabilitation: Reliability and Validity. Archives of Physical Medicine and Rehabilitation, 84, 1118-1124. http://dx.doi.org/10.1016/S0003-9993(03)00203-X

[7] Okahashi, S., Seki, K., Nagano, A., Luo, Z., Kojima, M. and Futaki, T. (2013) A Virtual Shopping Test for Realistic Assessment of Cognitive Function. Journal of NeuroEngineering and Rehabilitation, 10, 1, 59. http://www.jneuroengrehab.com/content/10/1/59

[8] Gagnon, L., Yucel, M.A., Dehaes, M., Cooper, R.J., Perdue, K.L., Selb, J., Huppert, T.J., Hoge, R.D. and Boas, D.A. (2012) Quantification of the Cortical Contribution to the NIRS Signal over the Motor Cortex Using Concurrent NIRS-fMRI Measurements. Neuroimage, 59, 3933-3940. http://dx.doi.org/10.1016/j.neuroimage.2011.10.054

[9] Villringer, A. and Chance, B. (1997) Non-Invasive Optical Spectroscopy and Imaging of Human Brain Function. Trends in Neurosciences, 20, 435-442. http://dx.doi.org/10.1016/S0166-2236(97)01132-6

[10] Miyai, I., Yagura, H., Hatakenaka, M., Oda, I., Konishi, I. and Kubota, K. (2003) Longitudinal Optical Imaging Study for Locomotor Recovery after Stroke. Stroke, 34, 2866-2870. http://dx.doi.org/10.1161/01.STR.0000100166.81077.8A

[11] Moriguchi, Y. and Hiraki, K. (2013) Prefrontal Cortex and Executive Function in Young Children: A Review of Nirsstudies. Frontiers in Human Neuroscience, 7, 867.

[12] Godefroy, O. (2003) Frontal Syndrome and Disorders of Executive Functions. Journal of Neurology, 250, 1-6. http://dx.doi.org/10.1007/s00415-003-0918-2

[13] Smith, A. (1982) Symbol Digit Modalities Test: Manual. Western Psychological Services, Los Angeles.

[14] Beck, L.H., Bransome Jr., E.D., Mirsky, A.F., Rosvold, H.E. and Sarason, I. (1956) A Continuous Performance Test of Brain Damage. J Consult Psychol, 20, 343-350. http://dx.doi.org/10.1037/h0043220

[15] Ishiai, S. (1999) Behavioural Inattention Test. Japanese Edition, Shinkoh Igaku Shuppan Co., Ltd., Tokyo.

[16] Wilson, B.A., Cockburn, J. and Baddeley, A. (1985) Rivermead Behavioural Memory Test. Thames Valley Test Company, London.

[17] Kazui, H., Hirono, N., Hashimoto, M., Nakano, Y., Matsumoto, K., Takatsuki, Y., Mori, E., Ikejiri, Y. and Takeda, M. (2006) Symptoms Underlying Unawareness of Memory Impairment in Patients with Mild Alzheimer's Disease. Journal of Geriatric Psychiatry and Neurology, 19, 3-12. http://dx.doi.org/10.1177/0891988705277543

[18] Wilson, B.A., Alderman, N., Burgess, P., Emslie, H. and Evans, J. (1966) Behavioural Assessment of the Dysexecutive Syndrome: Test Manual. Thames Valley Test Company, London.

[19] Toichi, M., Findling, R.L., Kubota, Y., Calabrese, J.R., Wiznitzer, M., McNamara, N.K. and Yamamoto, K. (2004) Hemodynamic Differences in the Activation of the Prefrontal Cortex: Attention vs. Higher Cognitive Processing. Neuropsychologia, 42, 698-706. http://dx.doi.org/10.1016/j.neuropsychologia.2003.08.012 
Scientific Research Publishing (SCIRP) is one of the largest Open Access journal publishers. It is currently publishing more than 200 open access, online, peer-reviewed journals covering a wide range of academic disciplines. SCIRP serves the worldwide academic communities and contributes to the progress and application of science with its publication.

Other selected journals from SCIRP are listed as below. Submit your manuscript to us via either submit@scirp.org or Online Submission Portal.
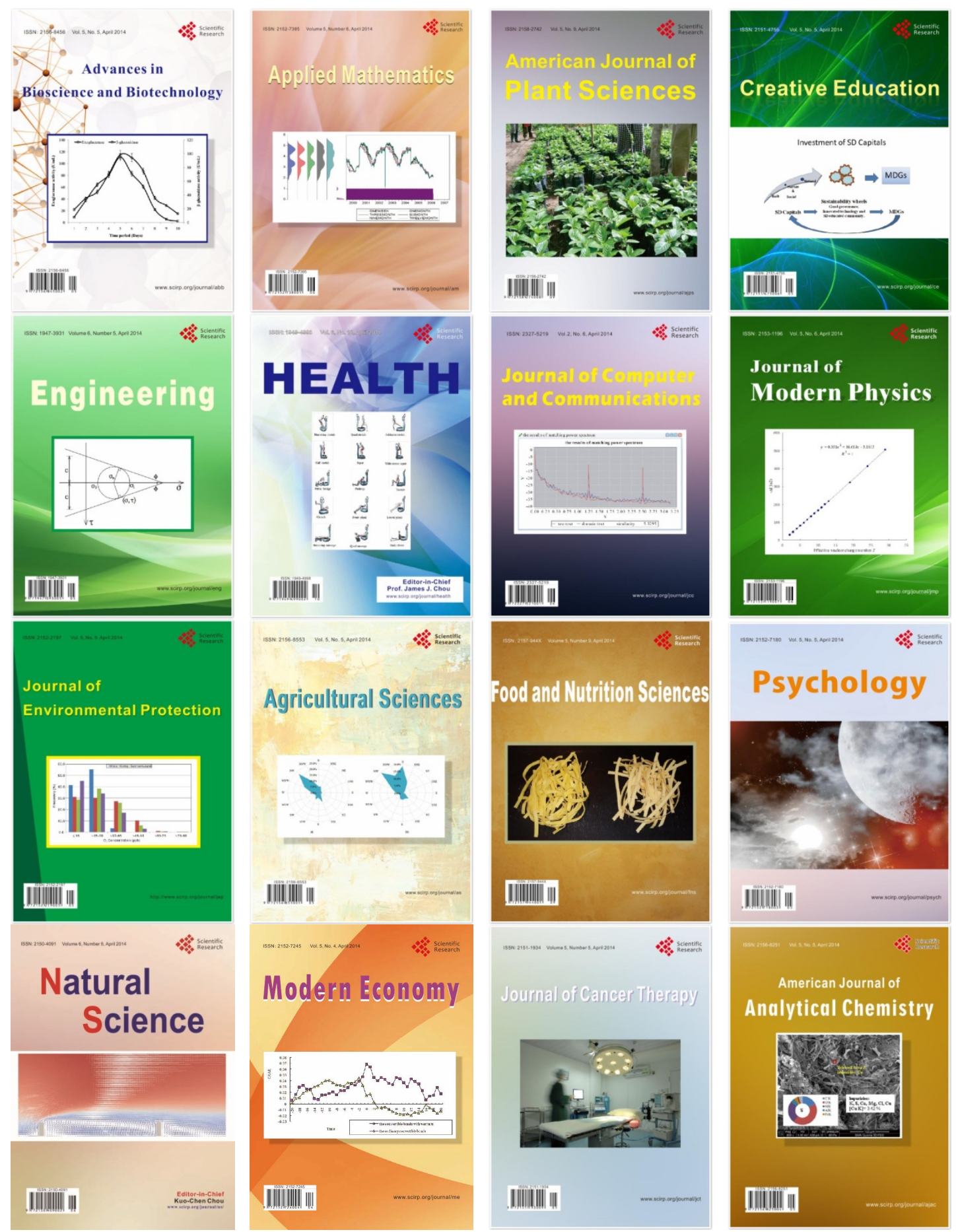